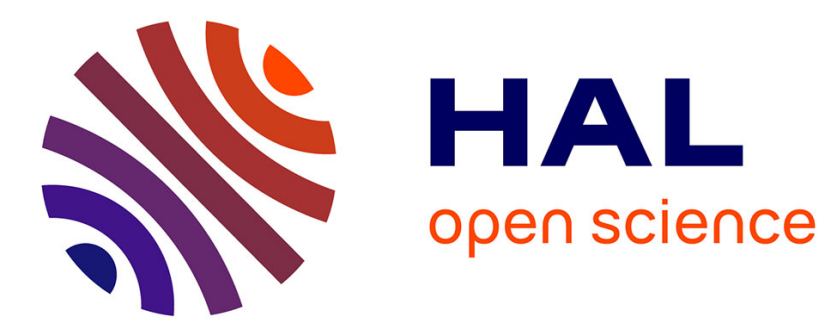

\title{
Theoretical studies of the tunneling splitting of malonaldehyde using the multiconfiguration time-dependent Hartree approach
}

\author{
Markus Schröder, Fabien Gatti, Hans-Dieter Meyer
}

\section{- To cite this version:}

Markus Schröder, Fabien Gatti, Hans-Dieter Meyer. Theoretical studies of the tunneling splitting of malonaldehyde using the multiconfiguration time-dependent Hartree approach. Journal of Chemical Physics, 2011, 134, pp.234307. 10.1063/1.3600343 . hal-00601672

HAL Id: hal-00601672

https://hal.science/hal-00601672

Submitted on 9 Jun 2021

HAL is a multi-disciplinary open access archive for the deposit and dissemination of scientific research documents, whether they are published or not. The documents may come from teaching and research institutions in France or abroad, or from public or private research centers.
L'archive ouverte pluridisciplinaire HAL, est destinée au dépôt et à la diffusion de documents scientifiques de niveau recherche, publiés ou non, émanant des établissements d'enseignement et de recherche français ou étrangers, des laboratoires publics ou privés. 


\title{
Theoretical studies of the tunneling splitting of malonaldehyde using the multiconfiguration time-dependent Hartree approach
}

\author{
Markus Schröder, ${ }^{1, a)}$ Fabien Gatti, ${ }^{2, b)}$ and Hans-Dieter Meyer ${ }^{1, c)}$ \\ ${ }^{1}$ Theoretische Chemie, Universität Heidelberg, Im Neuenheimer Feld 229, D-69120 Heidelberg, Germany \\ ${ }^{2}$ CTMM, Institut Gerhardt (UMR 5232-CNRS), CC 1501, Université de Montpellier II, \\ F-34095 Montpellier, Cedex 05, France
}

(Received 21 April 2011; accepted 25 May 2011; published online 17 June 2011)

\begin{abstract}
Full dimensional multi-configuration time-dependent Hartree calculations of the zero point energy and the tunneling splitting of malonaldehyde using a recently published potential energy surface [Y. Wang, B. J. Braams, J. M. Bowman, S. Carter, and D. P. Tew, J. Chem. Phys. 128, 224314 (2008)] are reported. The potential energy surface has been approximated by a modified version of the nmode representation and careful convergence check has been performed to ensure accurate results. The obtained value for the splitting $\left(23.4 \mathrm{~cm}^{-1}\right)$ is in acceptable agreement with the experimental value of $21.583 \mathrm{~cm}^{-1}$. The computed zero-point-energy is $14670 \mathrm{~cm}^{-1}$ which is lower than previous results of Wang et al., but likely to be about $4 \mathrm{~cm}^{-1}$ too low because of shortcomings of the nmode representation of the potential. The energies reported in this abstract contain a correction to account for neglected vibrational angular momentum terms. (C) 2011 American Institute of Physics. [doi:10.1063/1.3600343]
\end{abstract}

\section{INTRODUCTION}

Malonaldehyde is a prototype molecule to study intramolecular proton transfer processes that play an important role in many reactions of biological interest. Within malonaldehyde the transferred proton is located between two oxygen atoms which are connected by a chain of three carbon atoms with conjugated double bonds (saturated with hydrogen atoms). The carbon and oxygen atoms form the horseshoeshaped backbone of the molecule with the oxygen atoms at both ends. The molecule is planar within the equilibrium configuration and exhibits a $\mathrm{C}_{2 v}$ symmetry within the transition state. This property can be exploited for numerical calculations as has been done previously. ${ }^{1}$ The proton itself tunnels between the positions close to the oxygen atoms so that there exists a symmetric double well potential along the reaction path which gives rise to a tunneling splitting with an experimentally ${ }^{2,3}$ determined value of $21.583 \mathrm{~cm}^{-1}$.

Previous numerical studies on this system have been performed using a reaction surface description, ${ }^{4-6}$ more general methods, ${ }^{1,7-13}$ or semi-classical approaches. ${ }^{14-19}$ A full quantum mechanical treatment involving all 21 internal degrees of freedom remains a difficult task.

Recently, a full quantum mechanical calculation on malonaldehyde employing MCTDH has been reported ${ }^{1,8}$ using the potential energy surface of Yagi et al. ${ }^{20}$ However, it was very difficult for the authors to converge the splittings of the eigenstates. Our calculations confirm this. The correlation of the various degrees of freedom is very large, especially between the in-plane and the out-of-plane modes so that convergence is hard to achieve. As stated by the authors in the

\footnotetext{
a)Electronic mail: markus.schroeder@pci.uni-heidelberg.de.

b) Electronic mail: gatti@univ-montp2.fr.

c)Electronic mail: Hans-Dieter.Meyer@pci.uni-heidelberg.de.
}

conclusion of Ref. 1: "the present results show that the rigorous treatment of the 21-dimensional malonaldehyde system pushes the single-layer MCTDH approach used here to its limits."

In Ref. 1 as well as in the present contribution, a description in terms of normal coordinates is adopted. Clearly, the normal coordinates introduce strong artificial couplings, i.e., couplings introduced by using suboptimal coordinates. The Hydrogen transfer triggers a reorganization of the chemical bonds between the heavy backbone atoms, resulting in considerable shifts of the inter-atomic distances and bond angles such that the normal coordinates at the minimum geometries are quite different from the normal coordinates at the transition state.

Numerical studies show that the PES of Yagi et al. ${ }^{20}$ yields inversion splittings which are in reasonable agreement with the experiment. Following Wang et al. ${ }^{12}$ it does, however, not exhibit the correct barrier height which they determined to be approximately $4.1 \mathrm{kcal} / \mathrm{mol}$ using high level $a b$ initio calculations. In the present contribution we present results obtained with the multiconfiguration time-dependent Hartree (MCTDH) method and a recently published PES by Wang et al. ${ }^{12}$ Their PES exhibits a barrier height of $1438.1 \mathrm{~cm}^{-1}(=4.11 \mathrm{kcal} / \mathrm{mol})$. Wang and co-workers report (with an uncertainty of $\pm 2-3 \mathrm{~cm}^{-1}$ ) a ground state energy of $14678.3 \mathrm{~cm}^{-1}$ and an inversion splitting of $22.6 \mathrm{~cm}^{-1}$ obtained with a diffusion Monte-Carlo (DMC) calculation using normal mode coordinates after neglecting vibrationrotation coupling terms. With Cartesian coordinates they obtain a ground state energy of $14677.9 \mathrm{~cm}^{-1}$ and an inversion splitting of $21.6 \mathrm{~cm}^{-1}$, the latter being in excellent agreement with the experimental value ${ }^{2,3}$ of $21.583 \mathrm{~cm}^{-1}$, but this may be fortunate as the statistical DMC error is still a few $\mathrm{cm}^{-1}$.

The paper is organized as follows. In Sec. II the MCTDH algorithm is briefly reviewed while the model system and its 
representation is discussed in Sec. III. In Secs. IV and V the obtained results are presented and discussed, respectively.

\section{THE MULTICONFIGURATION TIME-DEPENDENT HARTREE METHOD}

MCTDH (Refs. 21-25) is a general method to solve the time-dependent as well as the time-independent Schrödinger equation. Within the MCTDH approach, the wavefunction $\Psi(Q, t)$ of the complete system is written as a sum of products of so-called single-particle functions (SPFs) $\varphi\left(Q_{\kappa}, t\right)$, which form a time-dependent and orthonormal basis set to represent the wavefunction of the system. The SPFs are lowdimensional functions, typically depending on one to three physical degrees of freedom (DOF). In cases, where the SPFs contain more than one DOF one introduces the composite or "combined" coordinate $Q_{\kappa}=\left\{q_{1, \kappa}, \ldots, q_{d_{\kappa}, \kappa}\right\}$ that comprises $d_{\kappa}$ physical degrees of freedom to form the particle $Q_{\kappa}$.

The ansatz for the MCTDH wavefunction reads

$$
\begin{aligned}
\Psi\left(q_{1}, \ldots, q_{f}, t\right) & \equiv \Psi\left(Q_{1}, \ldots, Q_{p}, t\right) \\
& =\sum_{j_{1}}^{n_{1}} \ldots \sum_{j_{p}}^{n_{p}} A_{j_{1}, \ldots, j_{p}}(t) \prod_{\kappa=1}^{p} \varphi_{j_{\kappa}}^{(\kappa)}\left(Q_{\kappa}, t\right) \\
& =\sum_{J} A_{J} \Phi_{J}
\end{aligned}
$$

where $f$ denotes the number of physical DOF, $p$ the number of particles, and $n_{\kappa}$ is the number of SPF for the $\kappa$ th (composite) coordinate. Furthermore, $A_{J} \equiv A_{j_{1} \ldots j_{p}}$ denote the MCTDH expansion coefficients of the total wavefunction within the given set of SPFs. The SPFs themselves are represented by linear combinations of time-independent primitive basis functions:

$$
\varphi_{j_{\kappa}}^{(\kappa)}\left(Q_{\kappa}, t\right)=\sum_{l_{1}=1}^{N_{1, \kappa}} \cdots \sum_{l_{d}=1}^{N_{d, \kappa}} c_{j_{\kappa} l_{1} \cdots l_{d}}^{(\kappa)}(t) \chi_{l_{1}}^{(\kappa)}\left(q_{1, \kappa}\right) \cdots \chi_{l_{d}}^{(\kappa)}\left(q_{d, \kappa}\right) .
$$

The primitive basis functions, $\chi$, are taken as discrete variable representation (DVR) functions, ${ }^{26,27}$ such that the SPFs are represented on a grid.

Applying the Dirac-Frenkel variational principle ${ }^{28,29}$ to the ansatz Eq. (1) leads then to equations of motion for the the expansion coefficients as well as the $\operatorname{SPF}(\hbar=1)$ :

$$
\begin{gathered}
i \frac{\partial}{\partial t} A_{J}=\sum_{L}\left\langle\Phi_{J}|\hat{H}| \Phi_{L}\right\rangle A_{L}, \\
i \frac{\partial}{\partial t} \varphi_{j}^{(\kappa)}=\left(1-P^{(\kappa)}\right) \sum_{k, l=1}^{n_{\kappa}}\left(\rho^{(\kappa)}\right)_{j, k}^{-1}\langle\hat{H}\rangle_{k, l}^{(\kappa)} \varphi_{l}^{(\kappa)},
\end{gathered}
$$

with $\hat{H}$ being the Hamiltonian of the system, $P^{(\kappa)}$ a projector on the subspace spanned by the SPF of the $\kappa$ th particle and $\rho^{(\kappa)}$ the single-particle density matrix. Furthermore, $\langle\hat{H}\rangle_{k, l}^{(\kappa)}$ are the so-called mean-field operators (cf., e.g., Refs. 22 or 23 ).

The evaluation of Eqs. (3) and (4), therefore, requires the calculation of multi-dimensional integrals over all DOF, involving the Hamiltonian operator $H$, at every time-step (cf. Ref. 23 for a detailed discussion). Similar to the MCTDH ansatz, Eq. (1), it is therefore helpful to express highdimensional terms within the Hamiltonian as a sum of products of low-dimensional ones. If the Hamiltonian is of product form

$$
\hat{H}=\sum_{r=1}^{s} c_{r} \prod_{\kappa=1}^{p} \hat{h}_{r}^{(\kappa)}
$$

with $\hat{h}_{r}^{(\kappa)}$ operating exclusively on the $\kappa$ th particle and $c_{r}$ being scalar coefficients, then the matrix elements of the Hamiltonian can be expressed by a sum of products of monomode integrals. For instance,

$$
\left\langle\Phi_{J}|\hat{H}| \Phi_{L}\right\rangle=\sum_{r=1}^{s} c_{r} \prod_{\kappa=1}^{p}\left\langle\varphi_{j_{\kappa}}\left|\hat{h}_{r}^{(\kappa)}\right| \varphi_{l_{\kappa}}\right\rangle,
$$

and similar expressions hold for the mean-field operators $\langle\hat{H}\rangle_{k, l}^{(\kappa)}$. If the number of terms in the Hamiltonian operator, $s$, is not too large, the numerical speed-up can be very substantial (see, e.g., Ref. 30 for a brief discussion). An alternative to this algorithm is, e.g., the CDVR method of U. Manthe. ${ }^{31}$

While the KEO often satisfies Eq. (5), potential energy surfaces (PES) are usually given as a complicated, nonseparable analytic expression. In this case, the PES cannot be used directly but has to be converted into a product form. If the combined primitive basis is not too large, i.e., below $\approx 10^{9}$ grid points, one may use the potfit algorithm ${ }^{23,25,32-34}$ to expand the potential into the desired product form. For larger grids, this is no longer possible so that a different approach has to be used. In the present, case a modification of the nmode-representation, also known as the cut-HDMR, ${ }^{35-42}$ has been used. We call this approach cluster expansion (CE). See Subsection III C for details.

\section{A. Improved relaxation}

The calculation of (low lying) eigenstates of the Hamiltonian is achieved by minimizing the expectation value of the Hamilton operator while keeping the $A$-(coefficient)-vectors as well as the SPF orthonormal. ${ }^{43-45}$ This leads to expressions for the A-vector:

$$
\sum_{L}\left\langle\Phi_{J}|\hat{H}| \Phi_{L}\right\rangle A_{L}=E A_{J}
$$

And the SPF:

$$
\sum_{l=1}^{n_{\kappa}}\langle\mathbf{H}\rangle_{j l}^{(\kappa)} \varphi_{l}^{(\kappa)}=\sum_{l=1}^{n_{\kappa}} \epsilon_{j l}^{(\kappa)} \varphi_{l}^{(\kappa)} .
$$

Equation (8) can be reformulated as a propagation in negative imaginary time $\tau=-i t$, Eq. (9), until relaxation is achieved:

$$
\frac{\partial}{\partial \tau} \varphi_{j}^{(\kappa)}:=-\left(1-P^{(\kappa)}\right) \sum_{k, l=1}^{n_{\kappa}}\left(\rho^{(\kappa)}\right)_{j k}^{-1}\langle\hat{H}\rangle_{k l}^{(\kappa)} \varphi_{l}^{(\kappa)}=0 .
$$

Convergence of an eigenstate is achieved if both, Eqs. (7) and (9), are fulfilled simultaneously. It is therefore straightforward to use an iterative algorithm to obtain converged eigenstates. One first chooses an initial guess state which presumably has a reasonable overlap with the desired eigenstate. Within the SPF basis functions of this state, Eq. (7) is solved using a 
Krylov-space method, typically a Davidson diagonalization, followed by selection of the desired eigenstate, calculation of the mean-fields and relaxation of the SPF. This diagonalization/relaxation procedure ${ }^{44,45}$ is then repeated until convergence is achieved.

\section{MODEL DESCRIPTION AND SYSTEM HAMILTONIAN}

Figure 1 depicts the positions of the nuclei of malonaldehyde at the "left" minimum (a), at the transition state (b) and at the "right" minimum (c). Already in this picture it can be seen that in particular the O-O distance and the shape of the backbone change substantially depending on the position of the transferred proton due to the reorganization of the single and double bonds. At the transition state the molecule exhibits $C_{2 v}$ symmetry - which will be made use of in Subsection III A.

It is in particular the large amplitude motion of the proton and the reorganization of bond lengths which makes an accurate description of the molecule rather difficult as a set of suited coordinates to describe the system has to be found. In addition, according to our calculations, the bending modes of all the $\mathrm{CH}$ bonds (in-plane and out-of-plane modes) are also strongly coupled with the reaction coordinate leading to the proton transfer. This result might appear counter intuitive since it is reasonable to think that the $\mathrm{CH}$ bonds do not play a major role in the process. Recently, we observed the same trend in the ring-opening of pyran. ${ }^{46}$ This coupling is due to the change of hybridization of the orbitals in the molecule. At the equilibrium geometry, the carbon atoms are $\mathrm{sp}^{2}$ hybridized but during the proton transfer the $\mathrm{sp}^{2}$ hybridized orbitals disappear and the $\mathrm{CH}$ bonds can almost freely wag (the corresponding frequencies of vibration of the bending modes become very small). After the proton transfer, the carbon atoms $\mathrm{sp}^{2}$-hybridize back. Consequently, the frequencies of the bending modes of vibration of the $\mathrm{CH}$ bonds strongly
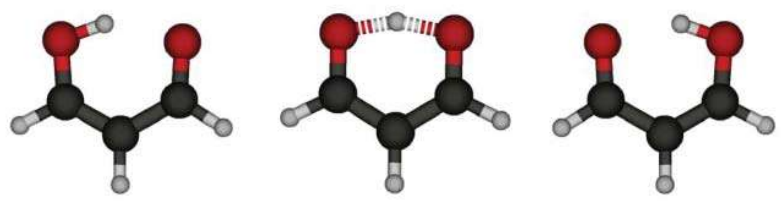<smiles>O=CC=CO</smiles>

(a)<smiles>O=CC=CO</smiles>

(c)
FIG. 1. Structure of malonaldehyde with the proton in the "left" minimum configuration (a), at the transition state (b), and in the "right" minimum configuration (c). Note, that the single- and double-bonds are interchanged after the proton transfer.
TABLE I. Numbering, frequencies, type and physical interpretation of the normal mode coordinates obtained at the transition state. The in-plane modes are of $A_{1}$ or $B_{2}$ symmetry, out-of-plane modes are of $A_{2}$ or $B_{1}$ symmetry. The modes which are symmetric with respect to the $q_{21}=0$ plane are of $A_{1}$ or $B_{1}$ and the ungerade of $A_{2}$ or $B_{2}$ symmetry. All energies are in $\mathrm{cm}^{-1}$.

\begin{tabular}{lrll}
\hline \hline Mode & Frequency & $C_{2 v}$ & \multicolumn{1}{c}{ Description } \\
\hline 1 & 346.28 & $A_{2}$ & CH-O bending \\
2 & 393.37 & $B_{1}$ & CH-C-CH bending \\
3 & 573.02 & $B_{2}$ & Ring deformation, C-O bending \\
4 & 608.85 & $A_{1}$ & Ring deformation, O-O stretching \\
5 & 750.20 & $B_{1}$ & C-H bending \\
6 & 957.92 & $A_{1}$ & Ring deformation, C-O bending \\
7 & 995.20 & $B_{1}$ & C-C-C bending \\
8 & 998.18 & $A_{2}$ & C-H bending \\
9 & 1073.78 & $A_{1}$ & Ring deformation, C-C stretching \\
10 & 1101.40 & $B_{2}$ & C-H bending \\
11 & 1321.98 & $B_{1}$ & Transfer-proton out-of-plane \\
12 & 1340.42 & $B_{2}$ & C-H bending \\
13 & 1405.12 & $A_{1}$ & C-H bending \\
14 & 1472.48 & $B_{2}$ & C-H bending \\
15 & 1617.71 & $A_{1}$ & C-O stretching \\
16 & 1620.12 & $B_{2}$ & C-C-C stretching \\
17 & 1893.66 & $A_{1}$ & Transfer-proton to ring center \\
18 & 3126.86 & $A_{1}$ & C-H stretching \\
19 & 3141.39 & $B_{2}$ & C-H stretching \\
20 & 3227.45 & $A_{1}$ & C-H stretching \\
21 & $\times 1253.02$ & $B_{2}$ & Proton transfer \\
\hline \hline
\end{tabular}

depend on the position of the proton and this is why one observes this correlation although malonaldehyde remains planar along the reaction path.

In the following we use the set of coordinates based on the normal modes obtained at the transition state. The proton transfer coordinate is automatically identified and due to the symmetry of the system, both minima are represented with equal quality. This representation, however, is not optimal at the two minima where the wavefunction will be mainly located. In Subsection III A, we briefly discuss how arising artificial correlations can be reduced by a simple coordinate shift. We further discuss there how the symmetry of the system can be exploited to further optimize a rendering of the system.

Throughout the paper we set the masses for Hydrogen to 1.0078, for Carbon to 12, and for Oxygen to 15.9949 atomic mass units (AMU) with one AMU equal to 1822.88848325 electron masses. Table I shows the normal mode frequencies $\omega_{i}$ obtained at the transition state with the aforementioned masses and symmetry properties of the normal vibrations.

\section{A. Kinetic energy operator}

As mentioned above, a description in normal mode coordinates $\tilde{q}_{i}$ introduces large artificial correlations between the different DOF. To minimize these effects we follow Hammer et al. ${ }^{1}$ and transform the coordinates according to

$$
\begin{aligned}
& q_{i}=\tilde{q}_{i}-F_{i}\left(\tilde{q}_{21}\right), \quad i=1 \ldots 20, \\
& q_{21}=\tilde{q}_{21},
\end{aligned}
$$

where the $F_{i}$ where obtained such that they resemble the reaction path of the proton transfer from the transition state to 
the two minima. This is done by minimizing the PES depending on the transfer coordinate $q_{21}$ and subsequent fitting of the obtained coordinate values to polynomials of 8th order for symmetric and of 7 th order for asymmetric $F_{i}$, respectively. The transformation equation (10) reduces the potential coupling and allows to use shorter grids. For the out-of-plane modes, the $F_{i}$ are set to zero because the molecule remains planar along the reaction path. But as already discussed, the out-of-plane motion couples strongly to the proton transfer motion as the out-of-plane frequencies change substantially along the reaction path. The out-of-plane bendings are rather poorly described by the rectilinear normal mode coordinates and curvilinear coordinates, e.g., bond angles would describe this motion much better. The use of normal node coordinates hence introduces artificial correlation which makes the convergence of the out-of-plane motion harder than expected.

Using the transformation equation (10), the kinetic energy operator $\hat{T}$ reads (after separating global rotation and translation as well as neglecting vibration-rotation-coupling terms)

$$
\begin{aligned}
\hat{T}= & -\frac{1}{2}\left[\sum_{i=1}^{21} \omega_{i} \frac{\partial^{2}}{\partial q_{21}^{2}}+\omega_{21} \sum_{i, j=1}^{20} F_{i}^{\prime}\left(q_{21}\right) F_{j}^{\prime}\left(q_{21}\right) \frac{\partial^{2}}{\partial q_{i} \partial q_{j}}\right. \\
& \left.-\omega_{21} \sum_{i=1}^{20}\left(F_{i}^{\prime}\left(q_{21}\right) \frac{\partial}{\partial q_{21}}+\frac{\partial}{\partial q_{21}} F_{i}^{\prime}\left(q_{21}\right)\right) \frac{\partial}{\partial q_{i}}\right],
\end{aligned}
$$

where $F_{i}^{\prime}\left(q_{21}\right)=\left(\partial / \partial q_{21}\right) F_{i}\left(q_{21}\right)$ denotes the first derivative of $F_{i}$ with respect to the transfer coordinate and $\omega_{i}$ the normal mode frequencies. Note that we use mass and frequency scaled - and hence dimensionless - normal mode coordinates and $\omega_{21}$ denotes the absolute value of the imaginary transition state frequency.

\section{B. Double well potentials}

For double well systems with a rather small tunneling rate it suggests itself to treat the two wells (almost) separately. ${ }^{1}$ To this end we introduce the projection operators

$$
\begin{aligned}
& h(x)= \begin{cases}1 & \text { if } x \geq 0, \\
0 & \text { otherwise, }\end{cases} \\
& h^{\prime}(x)=1-h(x),
\end{aligned}
$$

where $x$ denotes the reaction coordinate and $x=0$ the saddle point (i.e., $x=q_{21}$ for malonaldehyde). Next, we partition Hamiltonian and wavefunction

$$
\begin{aligned}
& \hat{H}=h \hat{H} h+h \hat{H} h^{\prime}+h^{\prime} \hat{H} h+h^{\prime} \hat{H} h^{\prime}, \\
& |\Psi\rangle=h|\Psi\rangle+h^{\prime}|\Psi\rangle=\left|\Psi_{1}\right\rangle+\left|\Psi_{2}\right\rangle .
\end{aligned}
$$

As $\left\langle\Psi_{1} \mid \Psi_{2}\right\rangle=\left\langle\Psi\left|h h^{\prime}\right| \Psi\right\rangle=0$ one may treat $\left|\Psi_{1}\right\rangle$ and $\left|\Psi_{2}\right\rangle$ like component wave functions of artificial "electronic" states so that the matrix form of the Hamiltonian reads

$$
\underline{H}=\left(\begin{array}{ll}
h \hat{H} h & h \hat{T} h^{\prime} \\
h^{\prime} \hat{T} h & h^{\prime} \hat{H} h^{\prime}
\end{array}\right) .
$$

Here we have replaced $\hat{H}$ by $\hat{T}$ in the off-diagonal elements because $h V h^{\prime}=h^{\prime} V h=0$. It is clear that the equations
$\hat{H}|\Psi\rangle=E|\Psi\rangle$ and $\underline{H}\left(\left|\Psi_{1}\right\rangle,\left|\Psi_{2}\right\rangle\right)^{T}=E\left(\left|\Psi_{1}\right\rangle,\left|\Psi_{2}\right\rangle\right)^{T}$ are strictly equivalent. However, the operators $h \hat{T} h, h \hat{T} h^{\prime}$, etc. are singular. Although the singularities cancel each other exactly, one may be concerned about the stability of a numerical treatment. Fortunately, using a grid representation turns the projected kinetic operators into ones which are bounded by $c / d^{2}$, where $c$ is a constant of order 1 and $d$ denotes the smallest distance between two grid points. Using a symmetric grid where $x=0$ lies in the middle between two grid points we found the numerics very stable.

The presented scheme is of advantage because a potential representation for state 1 (2) needs to be accurate in well 1 (2) only. However, a more significant advantage can be achieved if the double well is a symmetric double well. Following Hammer et al. ${ }^{1}$ we introduce the symmetry operator $R$ which is a reflection operator with respect to the plane $x=0$ :

$$
R \Psi\left(x, q_{1}, \ldots, q_{f-1}\right)=\Psi\left(-x, \alpha_{1} q_{1}, \ldots, \alpha_{f-1} q_{f-1}\right),
$$

with $\alpha_{i}= \pm 1$, depending on whether $q_{i}$ are "gerade" ( $A_{1}$ and $B_{1}$ for $\left.\mathrm{C}_{2 v}\right)$ or "ungerade" ( $A_{2}$ and $B_{2}$ for $\mathrm{C}_{2 v}$ ) modes with respect to the reflection. Obviously,

$$
R \Psi= \pm \Psi \text { and } R \Psi_{2}= \pm \Psi_{1}
$$

where "+" applies for "gerade" states, e.g., the ground state, and "-" to "ungerade" states, e.g., the first excited tunneling state.

Replacing $\Psi_{2}$ with $\pm R \Psi_{1}$ and using the first line of the Hamiltonian equation (15) only, leads to two uncoupled Schrödinger equations:

$$
\begin{aligned}
& H_{+}\left|\Psi_{+}\right\rangle=\left(h \hat{H} h+h \hat{T} h^{\prime} R\right)\left|\Psi_{+}\right\rangle=E_{+}\left|\Psi_{+}\right\rangle, \\
& H_{-}\left|\Psi_{-}\right\rangle=\left(h \hat{H} h-h \hat{T} h^{\prime} R\right)\left|\Psi_{-}\right\rangle=E_{-}\left|\Psi_{-}\right\rangle .
\end{aligned}
$$

Note, that $h \hat{T} h^{\prime} R=h \hat{T} R h$ and $[\hat{T}, R]=0$ holds from which follows that $H_{+}$and $H_{-}$are Hermitian. There is now a numerical gain as the number of grid points is halved and - more important for MCTDH - the number of SPF needed for convergence is smaller because only $\Psi_{1}$ but not $\Psi$ needs to be represented. The two solutions extended to the whole grid are orthonormal since they belong to different symmetry classes of the reflection operator (see Eq. (17)). The symmetry (and hence orthonormality) is strictly enforced by this approach.

\section{Potential energy surface and cluster expansion}

Once having defined a set of coordinates it still remains a challenging task to treat the PES within quantum dynamics calculations. A direct use of the full 21-dimensional PES of malonaldehyde is not possible due to the large size of the primitive grid. In order to reduce the amount of data that is needed to render the PES, a so-called cluster expansion (CE) or n-mode representation - has been used. ${ }^{36,37,39-41,47}$ Within the CE, the full 21-dimensional PES is approximated by nparticle interaction terms, where the particles are defined by combined modes as discussed above.

In the present case, we used a CE around one distinguished particle, $\tilde{Q}_{\text {ref }}$ which appears in all expansion terms, rather than a fixed reference point. ${ }^{12}$ One may call this a 
TABLE II. Mode combinations for cluster expansion.

\begin{tabular}{lc}
\hline \hline Logical coord. & Physical coord. \\
\hline $\bar{Q}_{1}$ & $q_{1}, q_{2}, q_{18}$ \\
$\bar{Q}_{2}$ & $q_{3}, q_{12}, q_{15}$ \\
$\bar{Q}_{3}$ & $q_{4}$ \\
$\bar{Q}_{4}$ & $q_{16}, q_{17}$ \\
$\bar{Q}_{5}$ & $q_{5}, q_{20}$ \\
$\bar{Q}_{6}$ & $q_{6}, q_{9}$ \\
$\bar{Q}_{7}$ & $q_{7}, q_{8}$ \\
$\bar{Q}_{8}$ & $q_{10}, q_{19}$ \\
$\bar{Q}_{9}$ & $q_{13}, q_{14}$ \\
$\bar{Q}_{10}=\bar{Q}_{\text {ref }}$ & $q_{11}, q_{21}$ \\
\hline \hline
\end{tabular}

reaction path $\mathrm{CE}$.

$$
\begin{aligned}
V(\overline{\mathbf{Q}})= & v_{\text {ref }}^{(0)}\left(\bar{Q}_{\text {ref }}\right)+\sum_{\alpha=1}^{p-1} v_{\alpha, \mathrm{ref}}^{(1)}\left(\bar{Q}_{\alpha}, \bar{Q}_{\text {ref }}\right) \\
& +\sum_{\alpha<\beta}^{p-1} v_{\alpha, \beta, \mathrm{ref}}^{(2)}\left(\bar{Q}_{\alpha}, \bar{Q}_{\beta}, \bar{Q}_{\mathrm{ref}}\right) \\
& +\sum_{\alpha<\beta<\gamma}^{p-1} v_{\alpha, \beta, \gamma, \mathrm{ref}}^{(3)}\left(\bar{Q}_{\alpha}, \bar{Q}_{\beta}, \bar{Q}_{\gamma}, \bar{Q}_{\mathrm{ref}}\right)+\cdots,
\end{aligned}
$$

where

$$
\begin{aligned}
v_{\text {ref }}^{(0)}\left(\bar{Q}_{\text {ref }}\right) & =V\left(\bar{Q}_{\text {ref }}, 0, \ldots, 0\right), \\
v_{\alpha, \text { ref }}^{(1)}\left(\bar{Q}_{\alpha}, \bar{Q}_{\text {ref }}\right) & =V\left(\bar{Q}_{\alpha}, \bar{Q}_{\text {ref }}, 0, \ldots, 0\right)-v_{\text {ref }}^{(0)}\left(\bar{Q}_{\text {ref }}\right),
\end{aligned}
$$

etc.

Naturally, for the proton transfer, $\bar{Q}_{\text {ref }}$ contains the reaction coordinate $q_{21}$ as it is of particular importance.

For the $\mathrm{CE}$ we introduce 10 particles or logical coordinates $\bar{Q}_{\kappa}, \kappa=1, \ldots, 10$, each comprising one to three physical coordinates as outlined in Table II. Here, it should be stressed that the combinations of coordinates given below are selected to provide a good convergence behavior of the CE. They have been chosen such that most of the largest correlations between two or three coordinates induced by the potential are already covered exactly within one particle $\bar{Q}_{\kappa}$ and higher order terms of Eq. (19) only add comparably small corrections while remaining (in terms of grid points) reasonably small, i.e., below $10^{9}$ sampling points on the combined primitive grid. The clusters (except $v^{(0)}$ ) are then brought to product form, Eq. (6), with the aid of the potfit algorithm. ${ }^{23,25,32-34}$

This mode combination for the $\mathrm{CE}$ differs from the one used for the actual MCTDH calculations as the $A$-vector in Eq. (1) would - for 10 particles - become too large to be treated efficiently. For the actual calculations within the MCTDH program, we further combined some of the logical coordinates outlined in Table II. The combination scheme used in MCTDH consists of 6 particles as displayed in Table III (see Subsection IV A).

As stated elsewhere, ${ }^{48}$ it is rather difficult to estimate at which order the expansion equation (19) can be truncated and which of the clusters are actually important for an accurate description of the PES, especially in the region where the
TABLE III. Mode combinations for MCTDH calculations.

\begin{tabular}{lcc}
\hline \hline Logical coord. (MCTDH) & Logical coord. (clusters) & Physical coords. \\
\hline$Q_{1}$ & $\bar{Q}_{1}$ & $q_{1}, q_{2}, q_{18}$ \\
$Q_{2}$ & $\bar{Q}_{2}$ & $q_{3}, q_{12}, q_{15}$ \\
$Q_{3}$ & $\bar{Q}_{3}, \bar{Q}_{4}$ & $q_{4}, q_{16}, q_{17}$ \\
$Q_{4}$ & $\bar{Q}_{5}, \bar{Q}_{6}$ & $q_{5}, q_{20}, q_{6}, q_{9}$ \\
$Q_{5}$ & $\bar{Q}_{7}, \bar{Q}_{8}$ & $q_{7}, q_{8}, q_{10}, q_{19}$ \\
$Q_{6}$ & $\bar{Q}_{9}, \bar{Q}_{10}$ & $q_{13}, q_{14}, q_{11}, q_{21}$ \\
\hline \hline
\end{tabular}

wavefunction resides. A natural choice would be an $\mathcal{L}^{2}$ norm, possibly subject to a weight function that roughly resembles the ground state density, to estimate the difference between the true and the approximated potential. However, this would require an integral over the complete grid which is precisely what is to be avoided. Also, the numerical effort that is needed to calculate the clusters prohibits a "brute force" generation of the terms in Eq. (19) beyond second or third order.

As in Ref. 48, we use a Metropolis algorithm ${ }^{49,50}$ to estimate the expectation values $M_{\alpha, \beta, \ldots}^{(m)}=\left\langle\left\langle v^{(m)}\left(\bar{Q}_{\alpha}\right.\right.\right.$, $\left.\left.\left.\bar{Q}_{\beta}, \ldots, \bar{Q}_{\text {ref }}\right)\right\rangle\right\rangle$ and root-mean-square (RMS) values $R_{\alpha, \beta, \ldots}^{(m)}$ $=\left\langle\left\langle v^{(m)}\left(\bar{Q}_{\alpha}, \bar{Q}_{\beta}, \ldots, \bar{Q}_{\text {ref }}\right)^{2}\right\rangle\right\rangle^{1 / 2}$ to estimate the contributions of the terms in Eq. (19). A similar analysis can be performed for the total error of all clusters compared to the exact PES.

Within the Metropolis algorithm, at step $i$ a random vector $\Delta \mathbf{q}$ of length $|\Delta \mathbf{q}|=l$ is generated and added the present coordinate vector $\mathbf{q}_{i}$ to generate the trial configuration $\mathbf{q}_{i}^{\prime}$ $=\mathbf{q}_{i}+\Delta \mathbf{q}$. The trial configuration is then tested against a random number $r$ in the interval $[0,1]$ via $p$ $=\exp \left(-\beta\left(V\left(\mathbf{q}_{i}^{\prime}\right)-V\left(\mathbf{q}_{i}\right)\right)\right)$ with $\beta$ being an "inverse temperature". If $r<p$ the step is accepted, leading to the new configuration $\mathbf{q}_{i+1}=\mathbf{q}_{i}^{\prime}$, otherwise the step is rejected and the procedure is repeated with a different random $\Delta \mathbf{q}$.

The trajectory generated by this Metropolis algorithm follows a Boltzmann distribution $(\alpha \exp (-\beta V))$ for the given temperature and roughly resembles the density of the ground state wavefunction. It can therefore be used to estimate the quality of the $\mathrm{CE}$ as well as the contributions of the single terms in Eq. (19). It should be noted here, however, that the width of the resulting Boltzmann distribution within the different DOF is only determined by the "temperature" and underlying potential. For a $1 \mathrm{D}$ harmonic potential the Boltzmann distribution $\exp (-\beta V)$ coincides with the ground state density if $\beta^{-1}=\omega / 2$. Hence, to achieve a distribution comparable with the ground state density in all DOF, a different temperature would have to be used for all DOF, scaled according to the normal mode frequencies as in Table I. This would account for the missing contribution of the KEO. As the system is not separable, this is not possible and choosing a single temperature therefore constitutes a compromise. In particular (and depending on the temperature chosen), the distribution will tend to be too wide for modes with low normal-mode frequencies and too narrow for modes with high normal-mode frequencies. In the present case a "temperature" $\beta^{-1}=400 \mathrm{~cm}^{-1}$ was used. 
As clusters we included the zeroth order one, $v^{(0)}\left(\bar{Q}_{\text {ref }}\right)$, and all first and second order clusters, $v_{i}^{(1)}\left(\bar{Q}_{i}, \bar{Q}_{\text {ref }}\right)$, $v_{i, j}^{(2)}\left(\bar{Q}_{i}, \bar{Q}_{j}, \bar{Q}_{\text {ref }}\right)$ with $1 \leq i \leq j \leq 9$. Guided by the statistical analysis, we additionally included the five clusters $v_{1,7,8}^{(3)}\left(\bar{Q}_{1}, q_{7}, \bar{Q}_{8}, \bar{Q}_{\text {ref }}\right), \quad v_{1,3,4}^{(3)}\left(\bar{Q}_{1}, \bar{Q}_{3}, \bar{Q}_{4}, q_{21}\right)$, $v_{2,3,4}^{(3)}\left(\bar{Q}_{2}, \bar{Q}_{3}, \bar{Q}_{4}, q_{21}\right), \quad v_{3,4,6}^{(3)}\left(\bar{Q}_{3}, \bar{Q}_{4}, \bar{Q}_{6}, \bar{Q}_{\mathrm{ref}}\right), \quad$ and $v_{4,5,6,8,9}^{(5)}\left(q_{9}, q_{10}, q_{11}, q_{14}, q_{16}, q_{20}\right)$ which are those that were found to have an expectation value $M$ of more then $\pm 1 \mathrm{~cm}^{-1}$. All other clusters have been neglected.

The amount of data reduction achieved by the $\mathrm{CE}$ is quite remarkable. The potential evaluated on the product grid consists of $1 \times 10^{23}$ data points, but the included clusters take only $1.7 \times 10^{8}$ points. After potfitting the clusters, the data are further reduced to $3 \times 10^{7}$ points.

\section{RESULTS}

\section{A. MCTDH setup}

As mentioned above, the particles used for the cluster expansion outlined in Table II have been further combined into six logical coordinates as shown in Table III to reduce the length of the $A$-vector and especially the size of the Hamiltonian matrix in the SPF basis.

Note, that the SPF now contains three to four physical DOF, thus reaching the limit of today's computational capacities. The underlying primitive grids for the physical coordinates are given in Table IV. For most of the normal mode coordinates 11-13 sampling points using a harmonic oscil-

TABLE IV. Primitive grids: HO - harmonic oscillator DVR; SIN - sine DVR functions using mass and frequency scaled normal modes. For the HO DVR the ranges result from the number of grid points chosen. As mass an frequency scaled normal modes are used, the mass and frequency parameters of the HO-DVR are set to unity.

\begin{tabular}{llcc}
\hline \hline $\begin{array}{l}\text { Physical } \\
\text { coordinate }\end{array}$ & $\begin{array}{c}\text { Type of } \\
\text { primitive basis }\end{array}$ & $\begin{array}{c}\text { No. of grid } \\
\text { points }\end{array}$ & Range \\
\hline$q_{1}$ & HO & 13 & -4.101 to 4.101 \\
$q_{2}$ & HO & 11 & -3.668 to 3.668 \\
$q_{3}$ & HO & 11 & -3.668 to 3.668 \\
$q_{4}$ & HO & 25 & -6.164 to 6.164 \\
$q_{5}$ & HO & 13 & -4.101 to 4.101 \\
$q_{6}$ & HO & 11 & -3.668 to 3.668 \\
$q_{7}$ & HO & 11 & -3.668 to 3.668 \\
$q_{8}$ & HO & 11 & -3.668 to 3.668 \\
$q_{9}$ & HO & 13 & -4.101 to 4.101 \\
$q_{10}$ & HO & 11 & -3.668 to 3.668 \\
$q_{11}$ & HO & 11 & -3.668 to 3.668 \\
$q_{12}$ & HO & 11 & -3.668 to 3.668 \\
$q_{13}$ & HO & 13 & -4.101 to 4.101 \\
$q_{14}$ & HO & 11 & -3.668 to 3.668 \\
$q_{15}$ & HO & 13 & -4.101 to 4.101 \\
$q_{16}$ & HO & 11 & -3.668 to 3.668 \\
$q_{17}$ & HO & 13 & -4.101 to 4.101 \\
$q_{18}$ & HO & 11 & -3.668 to 3.668 \\
$q_{19}$ & HO & 11 & -3.668 to 3.668 \\
$q_{20}$ & HO & 11 & -3.668 to 3.668 \\
$q_{21}$ & & 24 & -7.05 to -0.15 \\
\hline \hline
\end{tabular}

lator DVR proved to be sufficient for an accurate description, except for the mode $q_{4}$ and, naturally, $q_{21}$ which where sampled using 25 and 24 grid points, respectively. Note, that for $q_{21}$ a sine DVR is used and that it is only defined in one half of the complete range as the method outlined in Sec. III B is used.

\section{B. Convergence checks}

As discussed in Sec. II, the numerical effort for solving the Schrödinger equation within the MCTDH scheme crucially depends on the calculation of the mean fields and therefore the number (and size) of SPF used to represent the wavefunction. With exponentially increasing numerical effort, an accurate estimation of the state energy with a single calculation is therefore especially for large systems, such as malonaldehyde, not always possible. There are several measures to check the convergence of the MCTDH ansatz. In the following we use a scheme similar to the one proposed by Hammer and co-workers: ${ }^{1}$ starting from an in terms of the number of SPF reasonably well converged reference calculation, for each mode, an additional calculation is performed with the number of SPF doubled. Assuming that the change in energy for almost converged wavefunctions is independent of changes in other modes, the sum of all differences in energy to the reference is then added to the reference energy to give an estimate of the true state energy. Table V details the results obtained for the ground state and the first excited state for a wavefunction with a small set of SPF. Doubling the number of SPF leads to a large drop of the state energy especially for the particles 1 and 3. Extrapolating the state energy of the ground state and the first excited state leads to a zero point energy of $14669.424 \mathrm{~cm}^{-1}$ and a tunneling splitting of $23.563 \mathrm{~cm}^{-1}$.

Note, that - in contrast to the proposal of Hammer et al. ${ }^{1}$ - all results presented have been calculated using independent sets of SPF for the ground and excited state. This allows for a better convergence of the individual wavefunctions. If a combined set of SPF is used, the reference energy of the ground (first excited) state outlined in Table $\mathrm{V}$ is obtained as $14682.964(14707.750) \mathrm{cm}^{-1}$ which is $0.292(0.209) \mathrm{cm}^{-1}$ higher compared to calculations with separate sets of SPF. This is to be expected since in case of the combined set, the SPF constitute the basis set of two wave functions and cannot adopt optimally to either one of the wave functions. Since the convergence follows a variation principle higher energies indicate poorer convergence. On the other hand, the tunneling splitting is obtained as $24.786 \mathrm{~cm}^{-1}$, i.e., $0.083 \mathrm{~cm}^{-1}$ lower compared to the reference values of Table V. This may indicate that within a combined set of SPF the convergence of the excited state is slightly favored. However, the differences are small and they become smaller for better converged wave functions. Because separate calculations of $E_{+}$and $E_{-}$are numerically easier than a combined calculation and as the obtained ZPE is better, we prefer separate calculations.

Returning to the discussion of Table V, the large drop in energy in modes 1 and 3 suggests that the reference wavefunction was not well converged. Table VI outlines the result obtained for a reference state with larger sets of SPF. The 
TABLE V. Extrapolation of the state energies using a small set of SPF as reference. $\Delta E$ denotes the difference to the reference state. All energies are in $\mathrm{cm}^{-1}$.

\begin{tabular}{|c|c|c|c|c|c|c|c|c|c|c|}
\hline \multicolumn{6}{|c|}{ No. of SPF for } & \multirow[b]{2}{*}{ Energy $(+)$} & \multirow[b]{2}{*}{$\Delta E$} & \multirow[b]{2}{*}{ Energy (-) } & \multirow[b]{2}{*}{$\Delta E$} & \multirow[b]{2}{*}{ Splitting } \\
\hline$Q_{1}$ & $Q_{2}$ & $Q_{3}$ & $Q_{4}$ & $Q_{5}$ & $Q_{6}$ & & & & & \\
\hline 7 & 6 & 7 & 10 & 10 & 20 & 14682.672 & (reference) & 14707.541 & (reference) & 24.869 \\
\hline 14 & 6 & 7 & 10 & 10 & 20 & 14679.648 & -3.024 & 14704.377 & -3.164 & 24.729 \\
\hline 7 & 12 & 7 & 10 & 10 & 20 & 14681.712 & -0.960 & 14706.560 & -0.981 & 24.848 \\
\hline 7 & 6 & 14 & 10 & 10 & 20 & 14675.403 & -7.269 & 14699.317 & -8.224 & 23.914 \\
\hline 7 & 6 & 7 & 20 & 10 & 20 & 14682.071 & -0.601 & 14706.874 & -0.667 & 24.803 \\
\hline 7 & 6 & 7 & 10 & 20 & 20 & 14682.167 & -0.505 & 14707.061 & -0.480 & 24.894 \\
\hline \multirow[t]{3}{*}{7} & 6 & 7 & 10 & 10 & 40 & 14681.783 & -0.889 & 14706.503 & -1.038 & 24.720 \\
\hline & & & & & & Sum & -13.248 & & -14.554 & \\
\hline & & & & & \multicolumn{2}{|c|}{ Extrapolated energy } & 14669.424 & & 14692.987 & 23.563 \\
\hline
\end{tabular}

increased set of SPF led to a drop in energy of approximately $10 \mathrm{~cm}^{-1}$ for both, the ground and excited state.

Extrapolation of the reference energy leads to a reduction of additional $5 \mathrm{~cm}^{-1}$, with the largest contributions stemming from the increased number of SPF in modes three and six, leading altogether to an estimated inversion splitting of approximately $23.1 \mathrm{~cm}^{-1}$. Note, that for the reference calculations an inversion splitting of approximately $23.8 \mathrm{~cm}^{-1}$ is observed, i.e., the convergence of the MCTDH wavefunction seems to be of critical importance for result of the inversion splitting.

The results outlined in Table VI still reveal a large drop in energy upon increasing the number of SPF in modes three and six. Doubling the number of SPF in both modes simultaneously (last three rows of Table VI) leads to a ground state energy of $14669.709 \mathrm{~cm}^{-1}$ which is $3.075 \mathrm{~cm}^{-1}$ lower than the ZPE of obtained with the reference configuration. Interestingly, the drop in energy is $0.346 \mathrm{~cm}^{-1}(\approx 13 \%)$ larger than the sum of the two energy differences if the number of SPF in these modes is doubled independently from each other. This shows that the change in energy upon increasing the number of SPF in one mode is not independent of the number of SPF in other modes. If there are more SPFs in the other modes, the newly added SPFs allow for more correlation and an even larger drop in energy. The extrapolation scheme, therefore, does not violate the variational property, the extrapolated energies are still upper bounds to the exact ones.

Turning to the excited state, we note that we obtained with the same number of SPF a state energy of $14692.999 \mathrm{~cm}^{-1}$, i.e., $3.588 \mathrm{~cm}^{-1}$ lower than obtained with the reference configuration. Here, the drop in energy is $0.294 \mathrm{~cm}^{-1}(\approx 9 \%)$ larger than the sum of the individually obtained energy drops. Within this setup, therefore, the ZPE is obtained as $14667.314 \mathrm{~cm}^{-1}$ and the splitting as 23.201 $\mathrm{cm}^{-1}$, i.e., $0.052 \mathrm{~cm}^{-1}$ higher than estimated before.

To confirm the results outlined in the previous paragraph, additional calculations with increased numbers of SPF, especially in modes three and six has been performed as displayed in Table VII. Note that the first three rows are used for an extrapolation scheme as outlined above but for modes three and six only, where we increased the number of SPF only by $1 / 3$ due to limited computational capacities.

All direct comparisons in Table VII yield a splitting from 23.27 to $23.37 \mathrm{~cm}^{-1}$ and also the extrapolated value is $23.27 \mathrm{~cm}^{-1}$ which is quite consistent with the value obtained in the last line of Table VI. The drop in energy can mainly

TABLE VI. Extrapolation of the state energies as in Table V but with a larger set of SPF. $\Delta E$ denotes the difference to the reference state. The last three rows outline the extrapolation with the number of SPF increased in modes 3 and 6 simultaneously. We consider the values given in the last line of the table as our best results. All energies are in $\mathrm{cm}^{-1}$.

\begin{tabular}{|c|c|c|c|c|c|c|c|c|c|c|}
\hline \multicolumn{6}{|c|}{ No. of SPF for } & \multirow[b]{2}{*}{ Energy (+) } & \multirow[b]{2}{*}{$\Delta E$} & \multirow[b]{2}{*}{ Energy (-) } & \multirow[b]{2}{*}{$\Delta E$} & \multirow[b]{2}{*}{ Splitting } \\
\hline$Q_{1}$ & $Q_{2}$ & $Q_{3}$ & $Q_{4}$ & $Q_{5}$ & $Q_{6}$ & & & & & \\
\hline 10 & 7 & 12 & 10 & 10 & 20 & 14672.784 & (reference) & 14696.587 & (reference) & 23.803 \\
\hline 20 & 7 & 12 & 10 & 10 & 20 & 14672.273 & -0.511 & 14696.034 & -0.553 & 23.761 \\
\hline 10 & 14 & 12 & 10 & 10 & 20 & 14672.209 & -0.575 & 14695.985 & -0.602 & 23.776 \\
\hline 10 & 7 & 24 & 10 & 10 & 20 & 14671.321 & -1.463 & 14694.722 & -1.865 & 23.401 \\
\hline 10 & 7 & 12 & 20 & 10 & 20 & 14672.115 & -0.669 & 14695.847 & -0.740 & 23.732 \\
\hline 10 & 7 & 12 & 10 & 20 & 20 & 14672.144 & -0.640 & 14695.998 & -0.589 & 23.854 \\
\hline \multirow[t]{3}{*}{10} & 7 & 12 & 10 & 10 & 40 & 14671.518 & -1.266 & 14695.158 & -1.429 & 23.640 \\
\hline & & & & & & Sum & -5.124 & & -5.778 & \\
\hline & & & & & Extra & lated energy & 14667.660 & & 14690.809 & 23.149 \\
\hline \multirow[t]{3}{*}{10} & 7 & 24 & 10 & 10 & 40 & 14669.709 & -3.075 & 14692.999 & -3.588 & 23.290 \\
\hline & & & & & & Sum & -5.470 & & -6.072 & \\
\hline & & & & & Extra & lated energy & 14667.314 & & 14690.515 & 23.201 \\
\hline
\end{tabular}


TABLE VII. Largest direct calculations. The state energies are expected to lie slightly above the extrapolated ones reported in Table VI. All energies are in $\mathrm{cm}^{-1}$. The first three lines allow to extrapolate the energy. However, this extrapolation only includes modes $Q_{3}$ and $Q_{6}$. A full extrapolation including all modes is expected to lower the ZPE by additionally $0.3-0.6 \mathrm{~cm}^{-1}$, bringing it to very close agreement with the extrapolated values of Table VI. The last line of the table presents the results of our largest calculation.

\begin{tabular}{|c|c|c|c|c|c|c|c|c|}
\hline \multicolumn{6}{|c|}{ No. of SPF for } & \multirow[b]{2}{*}{ Energy $(+)$} & \multirow[b]{2}{*}{ Energy (-) } & \multirow[b]{2}{*}{ Splitting } \\
\hline$Q_{1}$ & $Q_{2}$ & $Q_{3}$ & $Q_{4}$ & $Q_{5}$ & $Q_{6}$ & & & \\
\hline 12 & 11 & 15 & 14 & 13 & 30 & 14669.066 & 14692.433 & 23.367 \\
\hline 12 & 11 & 20 & 14 & 13 & 30 & 14668.414 & 14691.737 & 23.323 \\
\hline \multirow[t]{2}{*}{12} & 11 & 15 & 14 & 13 & 40 & 14668.760 & 14692.076 & 23.316 \\
\hline & & \multicolumn{4}{|c|}{ Extrapolated energy } & 14668.108 & 14691.380 & 23.272 \\
\hline 13 & 11 & 20 & 15 & 14 & 33 & 14668.023 & 14691.293 & 23.270 \\
\hline
\end{tabular}

be ascribed to mode three while the contribution to the splitting is balanced. The largest calculations performed are outlined in the last line of Table VII. Here we obtain a ZPE of $14668.023 \mathrm{~cm}^{-1}$ and an inversion splitting of $23.270 \mathrm{~cm}^{-1}$ which is merely 0.7 and $0.07 \mathrm{~cm}^{-1}$ above the extrapolated values of Table VI.

\section{SUMMARY AND DISCUSSION}

Calculations of the full-dimensional symmetric and antisymmetric vibrational ground states of malonaldehyde have been performed to obtain the tunneling splitting of the proton transfer. Calculations including an extrapolation scheme yielded a ground state energy of $14667.3 \mathrm{~cm}^{-1}$ and a tunneling splitting of $23.2 \mathrm{~cm}^{-1}$, respectively. The following calculations with larger SPF basis sets, but without extrapolation, confirmed these results with an error of less than $2 \mathrm{~cm}^{-1}$ for the ground state and less than $0.1 \mathrm{~cm}^{-1}$ for the tunneling splitting. The extensive convergence checks show that the MCTDH energies are well converged. (See also the list of natural populations. ${ }^{52}$ ) Finally, when adding the estimated contributions of the vibration-rotation coupling terms ( 2.4 and $0.2 \mathrm{~cm}^{-1}$ obtained $^{51}$ as expectation values) to our results, we propose a ZPE of $14670 \mathrm{~cm}^{-1}$ and a tunneling splitting of $23.4 \mathrm{~cm}^{-1}$ for the used PES. ${ }^{12}$

The tunneling splitting obtained in the present calculations differs rather significantly from the experimental value of $21.6 \mathrm{~cm}^{-1}$. It does, however, confirm the inversion splitting of $22.6 \mathrm{~cm}^{-1}$ using normal mode coordinates reported in Ref. 12 within their stated error margins of $2-3 \mathrm{~cm}^{-1}$ and is in very good agreement with the splitting of $23.8 \mathrm{~cm}^{-1}$ recently obtained by Hammer and Manthe. ${ }^{51}$

The ZPE for normal mode coordinates, reported in Ref. 12 could not be reproduced. Within the present calculations it is estimated lower than stated by Wang et al., who reported a ZPE of $14678 \mathrm{~cm}^{-1}$. One source of error could be the truncation of the cluster expansion, and in fact we consider the MCTDH calculations as better converged than the potential representation. However, according to the statistical analysis we have performed, we consider it as unlikely that potential representation errors shift the ZPE down by more than $4 \mathrm{~cm}^{-1}$. The corresponding shift in the energy splitting is assumed an order of magnitude smaller because deficiencies of the potential representation affect the plus and the minus states in a similar way.

\section{ACKNOWLEDGMENTS}

The authors thank Professor J. Bowman for providing the potential energy routine for malonaldehyde and the Scientific Supercomputing Center Karlsruhe for providing computer time. The authors thank T. Hammer and U. Manthe for discussions and for letting us know their results prior to publication. Financial support through the German and French science foundations through contact DFG/ANR-09-BLA-0417 is also gratefully acknowledged.

${ }^{1}$ T. Hammer, M. D. Coutinho-Neto, A. Viel, and U. Manthe, J. Chem. Phys. 131, 224109 (2009)

${ }^{2}$ D. W. Firth, K. Beyer, M. A. Dvorak, S. W. Reeve, A. Grushow, and K. R. Leopold, J. Chem. Phys. 94, 1812 (1991).

${ }^{3}$ T. Baba, T. Tanaka, I. Morino, K. M.T. Yamada, and K. Tanaka, J. Chem. Phys. 110, 4131 (1999).

${ }^{4}$ T. Carrington, Jr. and W. H. Miller, J. Chem. Phys. 84, 4364 (1986).

${ }^{5}$ N. Shida, P. F. Barbara, and J. E. Almöf, J. Chem. Phys. 91, 4061 (1989)

${ }^{6}$ D. Tew, N. Handy, and S. Carter, J. Chem. Phys. 125, 084313 (2003).

${ }^{7}$ G. V. Mil'nikov, K. Yagi, T. Taketsugu, H. Nakamura, and K. Hirao, J. Chem. Phys. 120, 5036 (2004)

${ }^{8}$ M. D. Coutinho-Neto, A. Viel, and U. Manthe, J. Chem. Phys. 121, 9207 (2004).

${ }^{9}$ A. Viel, M. D. Coutinho-Neto, and U. Manthe, J. Chem. Phys. 126, 024308 (2007).

${ }^{10}$ A. Hazra, J. H. Skone, and S. Hammes-Schiffer, J. Chem. Phys. 130, 054108 (2009).

${ }^{11}$ Y. Wang and J. M. Bowman, J. Chem. Phys. 129, 121103 (2008).

${ }^{12}$ Y. Wang, B. J. Braams, J. M. Bowman, S. Carter, and D. P. Tew, J. Chem. Phys. 128, 224314 (2008).

${ }^{13}$ Y. Yang and M. Meuwly, J. Chem. Phys. 133, 064503 (2010).

${ }^{14}$ N. Makri and W. H. Miller, J. Chem. Phys. 91, 4026 (1989).

${ }^{15}$ Z. Smedarchina, W. Siebrand, and M. Z. Zgierski, J. Chem. Phys. 103, 5326 (1995).

${ }^{16}$ T. D. Sewell, Y. Guo, and D. L. Thompson, J. Chem. Phys. 103, 8557 (1995).

${ }^{17}$ M. Ben-Nun and T. J. Martínez, J. Phys. Chem. A 103, 6055 (1999).

${ }^{18}$ V. A. Benderskii, E. V. Vetoshkin, I. S. Irgibaeva, and H. P. Trommsdroff, Chem. Phys. 262, 393 (2000).

${ }^{19}$ M. E. Tuckermann and D. Marx, Phys. Rev. Lett. 86, 4946 (2010).

${ }^{20} \mathrm{~K}$. Yagi, T. Taketsugu, and K. Hirao, J. Chem. Phys. 115, 10647 (2001).

${ }^{21}$ H.-D. Meyer, U. Manthe, and L. S. Cederbaum, Chem. Phys. Lett. 165, 73 (1990).

${ }^{22}$ U. Manthe, H.-D. Meyer, and L. S. Cederbaum, J. Chem. Phys. 97, 3199 (1992).

${ }^{23}$ M. H. Beck, A. Jäckle, G. A. Worth, and H.-D. Meyer, Phys. Rep 324, 1 (2000).

${ }^{24}$ H.-D. Meyer and G. A. Worth, Theor. Chem. Acc. 109, 251 (2003).

${ }^{25}$ Multidimensional Quantum Dynamics: $M C T D H$ Theory and Applications, edited by H.-D. Meyer, F. Gatti, and G. A. Worth (Wiley-VCH, Weinheim, 2009).

${ }^{26} \mathrm{~J}$. C. Light, in Time-Dependent Quantum Molecular Dynamics, edited by J. Broeckhove and L. Lathouwers ( Plenum, New York, 1992), pp. 185-199.

${ }^{27}$ J. C. Light and T. Carrington, Jr., Adv. Chem. Phys. 114, 263 (2000).

${ }^{28}$ P. A.M. Dirac, Proc. Cambridge Philos. Soc. 26, 376 (1930).

${ }^{29}$ J. Frenkel, Wave Mechanics (Clarendon, Oxford, 1934).

${ }^{30}$ J. M. Bowman, T. Carrington, Jr., and H.-D. Meyer, Mol. Phys. 106, 2145 (2008).

${ }^{31}$ U. Manthe, J. Chem. Phys. 105, 6989 (1996)

${ }^{32}$ A. Jäckle and H.-D. Meyer, J. Chem. Phys. 104, 7974 (1996).

${ }^{33}$ A. Jäckle and H.-D. Meyer, J. Chem. Phys. 109, 3772 (1998).

${ }^{34}$ F. Gatti and H.-D. Meyer, Chem. Phys. 304, 3 (2004).

${ }^{35}$ S. Carter, S. J. Culik, and J. M. Bowman, J. Chem. Phys. 107, 10458 (1997). 
${ }^{36}$ H. Rabitz and O. F. Alis, J. Math. Chem. 25, 197 (1999).

${ }^{37}$ J. M. Bowman, S. Carter, and X. Huang, Int. Rev. Phys. Chem. 22, 533 (2003).

${ }^{38}$ G. Li, S. Wang, C. Rosenthal, and H. Rabitz, J. Math. Chem. 30, 1 (2001)

${ }^{39}$ O. F. Alis and H. Rabitz, J. Math. Chem. 29, 127 (2001).

${ }^{40}$ G. Y. Li, J. S. Hu, S. W. Wang, P. G. Georgopoulos, J. Schoendorf, and H. Rabitz, J. Phys. Chem. A 110, 2474 (2006).

${ }^{41}$ S. Manzhos and T. Carrington, J. Chem. Phys. 125, 084109 (2006).

${ }^{42}$ S. W. Wang, P. G. Georgopoulos, G. Y. Li, and H. Rabitz, J. Phys. Chem A 107, 4707 (2003)

${ }^{43}$ J. Hinze, J. Chem. Phys. 59, 6424 (1973).

${ }^{44}$ H.-D. Meyer, F. Le Quéré, C. Léonard, and F. Gatti, Chem. Phys. 329, 179 (2006)
${ }^{45}$ L. J. Doriol, F. Gatti, C. Iung, and H.-D. Meyer, J. Chem. Phys. 129, 224109 (2008).

${ }^{46}$ L. Joubert Doriol and F. Gatti (unpublished results)

${ }^{47}$ O. Vendrell, F. Gatti, D. Lauvergnat, and H.-D. Meyer, J. Chem. Phys. 127, 184302 (2007).

${ }^{48}$ O. Vendrell, M. Brill, F. Gatti, D. Lauvergnat, and H.-D. Meyer, J. Chem. Phys. 130, 234305 (2009).

${ }^{49}$ N. Metropolis, A. W. Rosenbluth, M. N. Rosenbluth, A. H. Teller, and E. Teller, J. Chem. Phys. 21, 1087 (1953).

${ }^{50}$ W. K. Hastings, Biometrika 57, 97 (1970).

${ }^{51}$ T. Hammer and U. Manthe, J. Chem. Phys. 134, 224305 (2011).

${ }^{52}$ See supplementary material at http://dx.doi.org/10.1063/1.3600343 for a list of natural populations. 